\title{
Quantum thermodynamic cooling cycle
}

\author{
José P. Palao* and Ronnie Kosloff \\ Department of Physical Chemistry and the Fritz Haber Research Center for Molecular Dynamics, \\ Hebrew University, Jerusalem 91904, Israel
}

\begin{abstract}
Jeffrey M. Gordon
Department of Energy and Environmental Physics, Blaustein Institute for Desert Research, Ben-Gurion University of the Negev, Sede Boqer Campus 84990, Israel and The Pearlstone Center for Aeronautical Engineering Studies, Department of Mechanical Engineering, Ben-Gurion University of the Negev, Beersheva 84105, Israel
\end{abstract}

\begin{abstract}
The quantum-mechanical and thermodynamic properties of a 3-level molecular cooling cycle are derived. An inadequacy of earlier models is rectified in accounting for the spontaneous emission and absorption associated with the coupling to the coherent driving field via an environmental reservoir. This additional coupling need not be dissipative, and can provide a thermal driving force - the quantum analog of classical absorption chillers. The dependence of the maximum attainable cooling rate on temperature, at ultra-low temperatures, is determined and shown to respect the recently-established fundamental bound based on the second and third laws of thermodynamics.
\end{abstract}

PACS number(s): 05.70.Ln, 32.80.Pj

\footnotetext{
*Also: Departamento de Física Fundamental II, Universidad de La Laguna, La Laguna 38204, Spain
} 


\section{INTRODUCTION}

Recently, a fundamental model-independent bound was derived for the temperature de-

pendence of the fastest rate $\dot{\mathcal{Q}}_{c}$ at which any substance can be cooled toward absolute zero via energetic exchange with a cold reservoir at temperature $T_{c}$ [四]:

$$
\dot{\mathcal{Q}}_{c} \propto T_{c}^{\alpha}, \quad \alpha \geq 1
$$

In the same study, a 3-level quantum-mechanical cooling cycle was postulated and shown to achieve this bound. The notion of analyzing molecular cooling with quantum thermodynamic cycles had been introduced earlier [2]. A system (working fluid) rejects heat to a hot bath, removes heat from a cold bath, and is driven by a coherent driving field: an idealized model for the laser cooling of magnetically-confined gases at ultra-low temperatures, as well as the laser cooling of solids and dyes [3, 田.

An important ingredient absent from previous models is the spontaneous emission and absorption related to the coupling between the system and the driving field. Superficially, this extra coupling provides a dissipative path and hence lowers achievable cooling rate. However, we will show that this coupling also introduces the possibility of driving the cooling cycle thermally, and broadens the conditions under which cooling can be generated. It constitutes the quantum-mechanical analog of classical absorption chillers. The quantum dynamics and thermodynamic properties of this improved 3-level model will be derived, in particular: (a) the identification of thermodynamic with quantum-mechanical variables; (b) the conditions required to produce cooling; (c) the dependence of cooling rate on the quantum control variables; (d) the efficiency of the cooling cycle; and (e) rigorous confirmation that the fundamental bound of Eq (1.1) is respected as the absolute zero is approached.

\section{THE 3-LEVEL MODEL}

The model is portrayed schematically in Fig 1: a 3-level system coupled to 3 infinite baths (reservoirs) plus an external driving field of coherent radiation. The allowed transitions are: 
(1) between levels 1 and 3 , with a hot bath at temperature $T_{h}$ (heat rejection); (2) between levels 1 and 2, with a cold bath at temperature $T_{c}$ (heat removal, i.e., cooling); and (3) between levels 2 and 3, simultaneously with the driving field and an environmental bath at temperature $T_{e}$ (dissipative heat rejection when $T_{e} \leq T_{h}$, and heat input when $T_{e}>T_{h}$ ). The environmental bath is treated as either: (a) independent of the hot and cold baths; or, when interference among the transitions is negligible [5], (b) representing an additional transition to the hot or cold bath. Two special cases are: (1) $T_{e}=T_{h}$, signifying spontaneous emission to the hot bath, i.e., coupling the transition to the hot bath; and (2) $T_{e}=T_{c}$, describing non-radiative (e.g., phonon) decay, i.e., coupling to the cold bath.

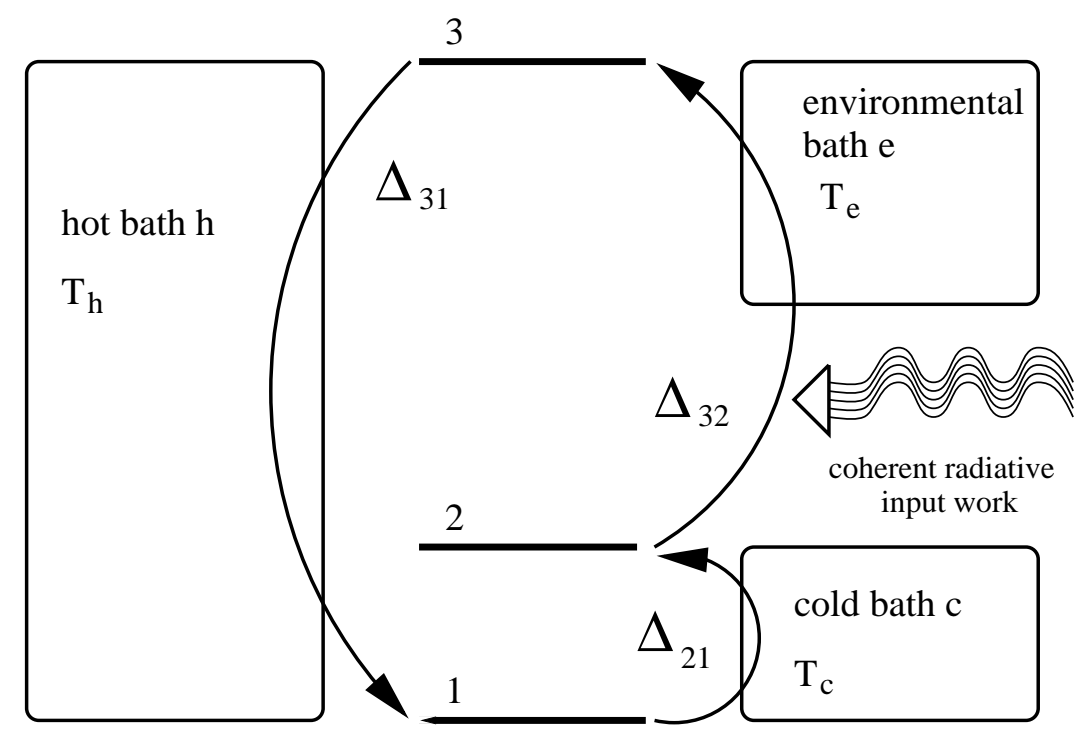

FIG. 1. Schematic of the 3-level quantum cooling cycle.

The Hamiltonian of the extended system (system, baths and driving field) is expressed as

$$
\hat{H}=\hat{H}_{s}+\hat{H}_{s f}(t)+\hat{H}_{s h}+\hat{H}_{s c}+\hat{H}_{s e}+\hat{H}_{h}+\hat{H}_{c}+\hat{H}_{e}
$$

where $\hat{H}_{s}$ is the Hamiltonian of the 3-level system (the working fluid of the thermodynamic cycle); $\hat{H}_{s f}(t)$ describes the time-dependent coupling between the system and the driving field (coherent radiation); $\hat{H}_{s h}, \hat{H}_{s c}, \hat{H}_{s e}$ account for the coupling between the system and 
its respective baths; and $\hat{H}_{h}, \hat{H}_{c}, \hat{H}_{e}$ are the respective Hamiltonians of the hot $(h)$, cold $(c)$ and environmental $(e)$ baths.

The system's Hamiltonian can be cast as

$$
\hat{H}_{s}=\Delta_{31} \hat{P}_{33}+\Delta_{21} \hat{P}_{22}
$$

where $\Delta_{i j}=E_{i}-E_{j}$ is the transition energy between levels $i$ and $j$ (with $E_{1}$ chosen as zero), and $\hat{P}_{i j}=|i\rangle\langle j|$ for $i=j$ are the projection operators over the states $i=1,2,3$. (Our units are chosen such that $\hbar=1$ and $k_{B}=1$.)

Our expression for $\hat{H}_{s f}(t)$ is based on the semi-classical rotating wave approximation [6]:

$$
\hat{H}_{s f}(t)=\epsilon\left(\hat{P}_{32} e^{-i \omega t}+\hat{P}_{23} e^{i \omega t}\right)
$$

for a field frequency $\omega$ and a coupling strength $\epsilon$ which depends on the amplitude of the driving field as well as the dipole moment of the transition, the latter being assumed to be independent of $\Delta_{32}$. With linear coupling between each transition in the 3-level system and its corresponding bath, the other coupling Hamiltonians can be written as:

$$
\begin{gathered}
\hat{H}_{s h}=\hat{P}_{31} \hat{\Gamma}_{h}+\hat{P}_{13} \hat{\Gamma}_{h}^{\dagger}, \\
\hat{H}_{s c}=\hat{P}_{21} \hat{\Gamma}_{c}+\hat{P}_{12} \hat{\Gamma}_{c}^{\dagger}, \\
\hat{H}_{s e}=\hat{P}_{32} \hat{\Gamma}_{e}+\hat{P}_{23} \hat{\Gamma}_{e}^{\dagger},
\end{gathered}
$$

where $\hat{P}_{i j}$ now represents the raising $(i>j)$ or lowering $(i<j)$ operator for the transition $i \leftrightarrow j$, and $\hat{\Gamma}_{b}$ denotes a bath operator $(b=h, c, e)$. Only the general properties of the bath correlation functions are needed to obtain the reduced description of the system's dynamics. The more detailed information on the bath Hamiltonians and the operators $\hat{\Gamma}_{b}$ will be derived afterwards when we examine the explicit forms of the correlation functions.

The standard tools for quantum open systems can be used to obtain a reduced description of system dynamics, i.e., the time evolution for the system's density matrix $\hat{\rho}_{s}(t)$, in terms of the operators of the 3-level system. $\hat{\rho}_{s}(t)=\operatorname{Tr}_{B}\{\hat{\rho}(t)\}$ where $\hat{\rho}$ is the density matrix of the extended system and $\operatorname{Tr}_{B}$ denotes the trace over all bath variables. We invoke the following approximations: 
- Weak coupling between the 3-level system and each bath.

- Factorization of the initial density matrix of the extended system,

$$
\hat{\rho}(t=0)=\hat{\rho}_{s}(t=0) \otimes \hat{\rho}_{h} \otimes \hat{\rho}_{c} \otimes \hat{\rho}_{e}
$$

with the initial density matrix of each bath assumed to be in thermal equilibrium,

$$
\hat{\rho}_{b}=\frac{e^{-\hat{H}_{b} / T_{b}}}{\operatorname{Tr}_{b}\left\{\hat{\rho}_{b}\right\}}
$$

- The Markovian assumption that the bath correlations

$$
C_{\hat{\Gamma}_{b} \hat{\Gamma}_{b}^{\prime}}=\operatorname{Tr}_{b}\left\{\hat{\Gamma}_{b} \hat{\Gamma}_{b}^{\prime}(-t) \hat{\rho}_{b}\right\}=\operatorname{Tr}_{b}\left\{\hat{\Gamma}_{b} e^{-i \hat{H}_{b} t} \hat{\Gamma}_{b}^{\prime} e^{i \hat{H}_{b} t} \hat{\rho}_{b}\right\}
$$

decay rapidly relative to the time scale of evolution of the 3-level system.

- Weak coupling with the driving field, i.e., $\epsilon$ being of the same order as the coupling with the baths. This approximation permits us to treat the effect of the field on the baths and the system separately [2,6].

These approximations result in the density matrix of the extended system factorizing at all times. One consequence is that the interfaces between the system and its baths become isothermal partitions which allow energy transfer but do not destroy the system's integrity, i.e., no quantum entanglement is created between the system and its baths [7]. This point is essential to the validity of the thermodynamic cycle approach, i.e., to distinct energy flows between a system and its reservoirs such that the intactness of the system is not compromised.

The analyses that follow build upon the derivation of the system's reduced dynamics as detailed in Appendix A. We adopt the Heisenberg representation and express the time evolution of an arbitrary operator $\hat{X}$ as

$$
\dot{\hat{X}}=i\left[\hat{H}_{s}+\hat{H}_{s f}(t), \hat{X}\right]+\left(\frac{\partial \hat{X}}{\partial t}\right)+\sum_{b=h, c, e} \mathcal{L}_{b}(\hat{X})
$$


where the super-operator $\mathcal{L}_{b}$ describes the effect of the baths on the dynamics of the 3-level system and possesses a structure (the Lindblad form) that assures the (complete) positivity of the reduced dynamics of Eq (2.8):

$$
\mathcal{L}_{b}(\hat{X})=\frac{\lambda_{b}}{2}\left\{\left[\hat{P}_{b}, \hat{X}\right] \hat{P}_{b}^{\dagger}-\hat{P}_{b}\left[\hat{P}_{b}^{\dagger}, \hat{X}\right]\right\}+\frac{\bar{\lambda}_{b}}{2}\left\{\left[\hat{P}_{b}^{\dagger}, \hat{X}\right] \hat{P}_{b}-\hat{P}_{b}^{\dagger}\left[\hat{P}_{b}, \hat{X}\right]\right\}
$$

with $\hat{P}_{b}=\hat{P}_{31}, \hat{P}_{21}, \hat{P}_{32}$ for $b=h, c, e$, repectively. The correlation coefficients $\lambda_{b}$ and $\bar{\lambda}_{b}$, defined in Appendix A, depend on the transition energies, the bath temperatures and the coupling strength. They appear as coefficients in the equations for $\hat{P}_{33}, \hat{P}_{22}, \hat{P}_{11}, \hat{P}_{32}$ and $\hat{P}_{23}$ that follow from Eq (2.8) and de-couple from the others:

$$
\begin{aligned}
& \dot{\hat{P}}_{33}=-i \epsilon e^{-i \omega t} \hat{P}_{32}+i \epsilon e^{i \omega t} \hat{P}_{23}-\left(\lambda_{h}+\lambda_{e}\right) \hat{P}_{33}+\bar{\lambda}_{e} \hat{P}_{22}+\bar{\lambda}_{h} \hat{P}_{11} \\
& \dot{\hat{P}}_{22}=i \epsilon e^{-i \omega t} \hat{P}_{32}-i \epsilon e^{i \omega t} \hat{P}_{23}+\lambda_{e} \hat{P}_{33}-\left(\lambda_{c}+\bar{\lambda}_{e}\right) \hat{P}_{22}+\bar{\lambda}_{c} \hat{P}_{11} \\
& \dot{\hat{P}}_{11}=\lambda_{h} \hat{P}_{33}+\lambda_{c} P_{22}+-\left(\bar{\lambda}_{h}+\bar{\lambda}_{c}\right) \hat{P}_{11} \\
& \dot{\hat{P}}_{32}=i \Delta_{32} \hat{P}_{32}-i \epsilon e^{i \omega t} \hat{P}_{33}+i \epsilon e^{i \omega t} \hat{P}_{22}-\frac{1}{2}\left\{\lambda_{h}+\lambda_{c}+\lambda_{e}+\bar{\lambda}_{e}\right\} P_{32} \\
& \hat{P}_{23}=\hat{P}_{32}^{\dagger} .
\end{aligned}
$$

The terms $\hat{P}_{i j}$ are related to the diagonal elements of the reduced density matrix when $i=j\left(\operatorname{Tr}_{s}\left\{\hat{P}_{i i} \hat{\rho}_{s}\right\}=\rho_{i i}\right)$, and to the off-diagonal elements when $i \neq j\left(\operatorname{Tr}_{s}\left\{P_{i j} \hat{\rho}_{s}\right\}=\rho_{j i}\right)$. Hence Eq (2.10) represents rate equations for the density matrix elements. The diagonal and off-diagonal elements are coupled by the driving field. The bath is responsible for the self-couplings of both diagonal and off-diagonal elements. $\lambda_{b}$ and $\bar{\lambda}_{b}$ are then transition probabilities, per unit time, between energy levels. For example, $\lambda_{h}$ is the transition probability of a decay from level 3 to 1 in which energy is rejected to the hot bath, and $\bar{\lambda}_{h}$ is the probability for an excitation from level 1 to 3 in which energy is absorbed from the hot bath.

\section{IDENTIFICATION OF THE THERMODYNAMIC VARIABLES}

The identity of the thermodynamic energy flows in the cooling cycle follows from energy conservation and averaging [8.9]. The Hamiltonian $\hat{H}_{T}=\hat{H}_{s}+\hat{H}_{s f}(t)$ is introduced into the 
evolution equation (2.8), is multiplied by the initial density matrix, and is traced over the variables of the 3-level system, to yield

$$
\left\langle\dot{\hat{H}}_{T}\right\rangle=\left\langle\frac{\partial \hat{H}_{s f}(t)}{\partial t}\right\rangle+\left\langle\mathcal{L}_{h}\left(\hat{H}_{T}\right)\right\rangle+\left\langle\mathcal{L}_{c}\left(\hat{H}_{T}\right)\right\rangle+\left\langle\mathcal{L}_{e}\left(\hat{H}_{T}\right)\right\rangle
$$

The energy flow associated with the driving field (the first term on the RHS of Eq (3.1)) is the power input (cycle-averaged work) to the cycle, $\dot{\mathcal{W}}$. The remaining 3 terms are the respective heat flows between the system and its baths, $\dot{\mathcal{Q}}_{h}, \dot{\mathcal{Q}}_{c}, \dot{\mathcal{Q}}_{e}$. At steady state, $\left\langle\hat{H}_{T}\right\rangle$ is constant and independent of the system's initial state, so $\left\langle\dot{\hat{H}}_{T}\right\rangle=0$ and Eq (3.1) can be expressed as the First Law of thermodynamics for the thermodynamic cycle:

$$
\dot{\mathcal{W}}+\dot{\mathcal{Q}}_{h}+\dot{\mathcal{Q}}_{c}+\dot{\mathcal{Q}}_{e}=0
$$

with energy flows into the system defined as positive.

Now we can derive the relation between the cycle's thermodynamic variables and the quantum-mechanical parameters. At steady state (denoted by the superscript $s s$ ), the general $P$ operators become

$$
\hat{P}_{i j} \rightarrow \hat{P}_{i j}^{s s} e^{i \alpha_{i j} t}
$$

where $\dot{\hat{P}}_{i j}^{s s}=0, \alpha_{i i}=0, \alpha_{32}=\omega$ and the remaining values of $\hat{P}_{i j}^{s s}$ follow from introducing (3.3) into $\mathrm{Eq}(2.10)$ and solving the resultant set of linear time-independent equations. The solutions for $\hat{P}_{i j}^{s s}$ turn out to be proportional to the identity operator $\hat{1}, \hat{P}_{i j}^{s s}=p_{i j} \hat{1}$, where $p_{i j}$ is real when $i=j$ and complex when $i \neq j$. From the relation between the $\hat{P}$ operators and the elements of the density matrix, the steady-state expectation values of the populations (i.e., the diagonal elements) are constants, $\left\langle\hat{P}_{i i}\right\rangle=\left\langle\hat{P}_{i i}^{s s}\right\rangle=p_{i i}$, and the expectation values of the off-diagonal elements $\hat{P}_{32}$ and $\hat{P}_{23}$ oscillate in time, $\left\langle\hat{P}_{i j}\right\rangle=\left\langle\hat{P}_{i j}^{s s} e^{i \alpha_{i j} t}\right\rangle=p_{i j} e^{i \alpha_{i j} t}$. Explicit formulae for $p_{i j}$ are provided in Appendix $\mathrm{B}$ for the condition of resonance that is assumed in the analysis that follows

$$
\omega \equiv \Delta_{32}
$$


The cycle-average thermodynamic variables of the quantum refrigerator can now be expressed as:

$$
\begin{aligned}
& \dot{\mathcal{W}}=2 \epsilon \Delta_{32} \operatorname{Imag}\left[p_{32}\right]=\Delta_{32} \epsilon^{2}\left\{A\left(n_{c}-n_{h}\right)+B\right\} \\
& \dot{\mathcal{Q}}_{h}=\Delta_{31}\left(-\lambda_{h} p_{33}+\bar{\lambda}_{h} p_{11}\right)=-\Delta_{31}\left\{\epsilon^{2} A\left(n_{c}-n_{h}\right)+C\left(n_{c} n_{e}-n_{h}\right)\right\} \\
& \dot{\mathcal{Q}}_{c}=\Delta_{21}\left(-\lambda_{c} p_{22}+\bar{\lambda}_{c} p_{11}\right)=\Delta_{21}\left\{\epsilon^{2} A\left(n_{c}-n_{h}\right)+C\left(n_{c} n_{e}-n_{h}\right)\right\} \\
& \dot{\mathcal{Q}}_{e}=\Delta_{32}\left(-\lambda_{e} p_{33}+\bar{\lambda}_{e} p_{22}\right)=-\Delta_{32}\left\{\epsilon^{2} B-C\left(n_{c} n_{e}-n_{h}\right)\right\}
\end{aligned}
$$

where Eq (A6) of Appendix A has been used, and $n_{b}$ denotes the equilibrium populations: $n_{h}=e^{-\Delta_{31} / T_{h}}, n_{c}=e^{-\Delta_{21} / T_{c}}, n_{e}=e^{-\Delta_{32} / T_{e}}$. Detailed expressions for the positive coefficients $A, B$ and $C$ are presented in Appendix B, and depend on the bath temperatures, the transition energies and the coupling strength. Each energy flow constitutes a competition between two processes with rates $\Delta_{b} \lambda_{b} p_{k k}$ and $\Delta_{b} \bar{\lambda}_{b} p_{l l}(k>l)$, with the former describing heat rejection of magnitude $\Delta_{b}$ to the bath in the decay $k \rightarrow l$, and the latter representing heat removal of the same magnitude from the bath in the excitation $l \rightarrow k$. Also, as will be elucidated in Section $\nabla \mathbb{1}$, the work input rate $\dot{\mathcal{W}}$ need not be the only driving force for the cooling cycle. When $T_{e}>T_{h}$, the incoherent thermal flow $\dot{\mathcal{Q}}_{e}$ can become positive and hence can also contribute to the cooling rate.

The entropy production rate for the cycle is

$$
\begin{aligned}
\dot{S} & =-\left\{\frac{\dot{\mathcal{Q}}_{h}}{T_{h}}+\frac{\dot{\mathcal{Q}}_{c}}{T_{c}}+\frac{\dot{\mathcal{Q}}_{e}}{T_{e}}\right\}= \\
& =-4 \epsilon^{2} \frac{\lambda_{c} \lambda_{h}}{D}\left(n_{c}-n_{h}\right)\left(\frac{\Delta_{21}}{T_{c}}-\frac{\Delta_{31}}{T_{h}}\right)+4 \epsilon^{2} \frac{\lambda_{e}\left(\bar{\lambda}_{c}+\bar{\lambda}_{h}\right)}{D} \frac{\Delta_{32}}{T_{e}}\left(1-n_{e}\right)+ \\
& -\frac{\lambda_{h} \lambda_{c} \lambda_{e}}{D}\left(n_{h}-n_{c} n_{e}\right)\left(\frac{\Delta_{31}}{T_{h}}-\frac{\Delta_{21}}{T_{c}}-\frac{\Delta_{32}}{T_{e}}\right)
\end{aligned}
$$

where $D$ is a positive function derived in Appendix $\mathrm{B}$. With the relations noted above for the equilibrium populations $n_{b}$, it is straightforward to prove that $\dot{S}$ in Eq (3.6) must be non-negative, in accordance with the Second Law. 


\section{COUPLING WITH THE BATHS}

The results for the cooling performance of the quantum refrigeration cycle depend on the particulars of the coupling between the system and its baths. Hence specific models must be invoked. The primary variable of interest is the cooling rate (the interaction with the cold bath). Since in any event the results that will now be derived are independent of the nature of the hot and environmental reservoirs, we will treat these two as white baths, i.e., baths with a constant density of energy modes (at least in the frequency range of interest), for which

$$
\lambda_{b}=\Lambda_{b}, \quad \bar{\lambda}_{b}=\Lambda_{b} e^{-\Delta_{b} / T_{b}}
$$

where $\Lambda_{b}$ is the strength of the coupling.

Earlier quantum refrigeration models also treated the cold bath as having a constant mode density [1:2]. Here a more elaborate and realistic model is introduced for the cold bath: an assembly of harmonic oscillators - a viable model in the weak coupling limit [10]. The bath's Hamiltonian can then be expressed as

$$
\hat{H}_{c}=\sum_{i} \Delta_{c i} \hat{a}_{c i}^{\dagger} \hat{a}_{c i}
$$

where $\hat{a}_{c i}$ and $\hat{a}_{c i}^{\dagger}$ are the annihilation and creation operators, with the index $i$ spanning the bath oscillator energies $\Delta_{c i}$. The operator of Eq (2.4), that couples the system and bath is then

$$
\hat{\Gamma}_{c}=\sum_{i} g_{c i} \hat{a}_{c i}+g_{c i}^{*} \hat{a}_{c i}^{\dagger}
$$

where $g_{c i}$ and $g_{c i}^{*}$ denote the coupling constants.

The influence of an harmonic bath on system dynamics is embedded in the spectral strength function [10,11] $J_{c}(\Delta)=\sum_{i}\left|g_{c i}\right|^{2} \delta\left(\Delta-\Delta_{c i}\right)$ which, for low energies, is well approximated by a simple power law dependence

$$
J_{c}(\Delta) \approx \Lambda_{c} \Delta^{s_{c}}
$$


where $s_{c}$ must be positive [12]. The coefficients $\lambda$ then follow as

$$
\lambda_{c}\left(\Delta_{c}, T_{c}\right)=\frac{\Lambda_{c} \Delta_{c}^{s_{c}}}{1-e^{-\Delta_{c} / T_{c}}}, \quad \bar{\lambda}_{c}\left(\Delta_{c}, T_{c}\right)=\frac{\Lambda_{c} \Delta_{c}^{s_{c}}}{e^{\Delta_{c} / T_{c}}-1} .
$$

\section{COOLING PERFORMANCE}

\section{A. Objectives}

Several aspects of the thermodynamic performance of the quantum cooling cycle will be addressed in this section: (1) how strong a coupling with the driving field is required to achieve cooling (i.e., to insure $\dot{\mathcal{Q}}_{c}>0$ ); (2) how does cooling rate vary with the natural control variable $\Delta_{21}$ and under what conditions is cooling rate maximized; (3) how does maximum cooling rate depend on $T_{c}$ in the limit of the absolute zero and how does this dependence compare to the fundamental bound established from the Second and Third Laws; and (4) what is the efficiency of this cooling cycle and can it be cast in the same form as classical chiller analyses. In this section, only $T_{e}$ values in the range $T_{c} \leq T_{e} \leq T_{h}$ are analyzed, so the environmental bath serves a solely dissipative role. The following section will focus on the special thermodynamic consequences when $T_{e}>T_{h}$.

\section{B. Cooling window and electronic analog}

Because of the competition between the coupling to the driving field and dissipative losses, only certain combinations of system parameters produce cooling. The thermoelectric chiller offers a familiar example of the existence of a refrigeration window, where the cooling effect exists only for: (a) a sufficiently high thermopower (Seebeck coefficient); and (b) a particular voltage window. There are analogous limits in the 3-level quantum chiller.

In fact, the 3-level model can be viewed as an electronic device, with the analog of the voltage $V$ being $\Delta_{32}$ (in units of electronic charge). The maximum voltage is $V_{\max }=\Delta_{31}$,

and $\Delta_{21}=V_{\max }-V$. The power input is the product of voltage and current, so that 
$\mathrm{Eq}(3.5)$ provides the analog of the electrical current as a complicated implicit function of the system parameters, as well the relation between current and voltage. In the absence of the environmental bath, current is an exponentially increasing function of voltage [1] which, not coincidentally, is the same as for ideal diodes. With the added dissipation to the environmental bath, the current remains a strongly increasing function of voltage, albeit not strictly exponential, similar to non-ideal diodes.

The minimum coupling strength with the driving field $\epsilon_{\min }$ to produce a cooling effect follows from $\mathrm{Eq}(3.5)$ :

$$
\epsilon_{\min }=\sqrt{C\left(n_{h}-n_{e}\right) /\left\{A\left(1-n_{h}\right)\right\}}
$$

and is plotted as a function of $T_{e}$ in Fig 2 .

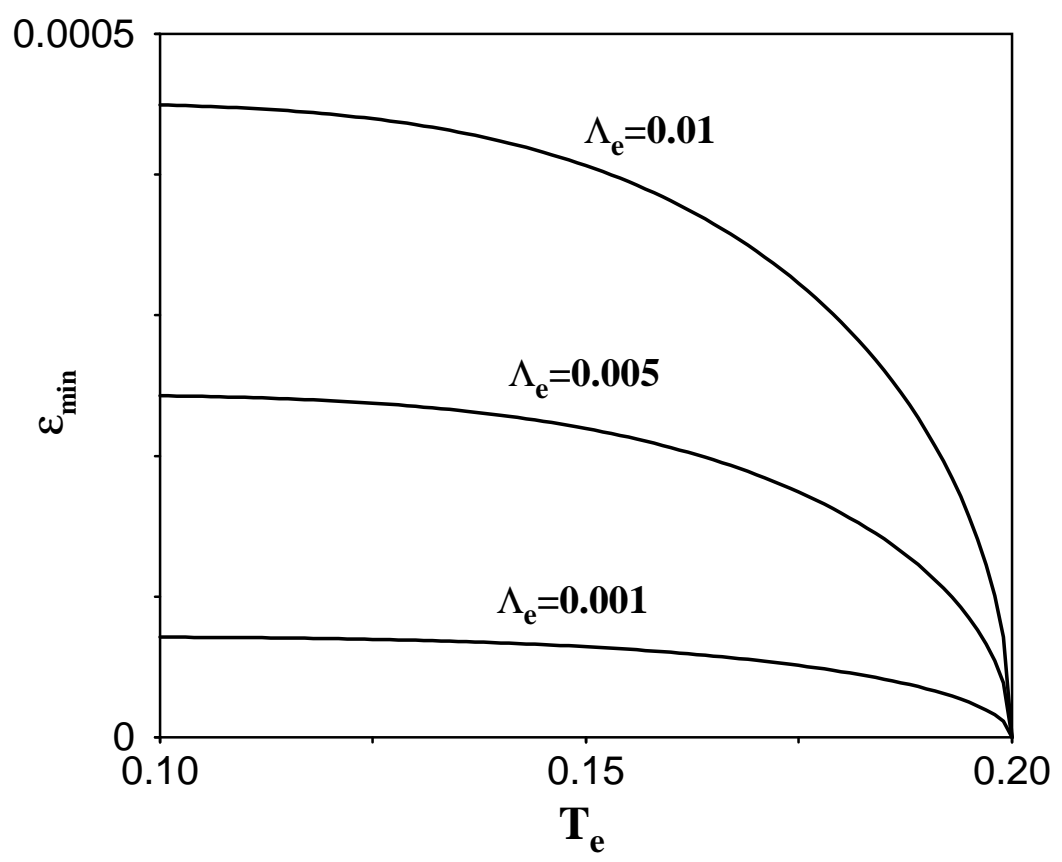

FIG. 2. Minimum coupling needed for cooling, as a function of the environmental bath temperature, at 3 values of coupling coefficient with the environmental bath. Other system parameters are fixed at: $T_{c}=0.1, T_{h}=0.2, \Delta_{31}=1, \Lambda_{h}=\Lambda_{c}=0.001$, and $s_{c}=1$. The regime of $T_{e}>T_{h}$, where no coupling is required to produce cooling, is addressed in Section V1. 


\section{Maximum cooling rate and the fundamental bound}

Our "control knob" for varying cooling rate is the transition energy $\Delta_{21}$. The cooling rate vanishes at two values of $\Delta_{21}$ that delimit the cooling window: $\Delta_{21}=0$ and $\Delta_{21}=\Delta_{21}^{\max }$, where $\Delta_{21}^{\max }$ depends on the principal system parameters, most notably $T_{c}$ and $\Lambda_{e}$ (Eq (3.5)), as illustrated in Fig 3 . Sample curves of cooling rate as a function of $\Delta_{21}$ are plotted in Fig 田.

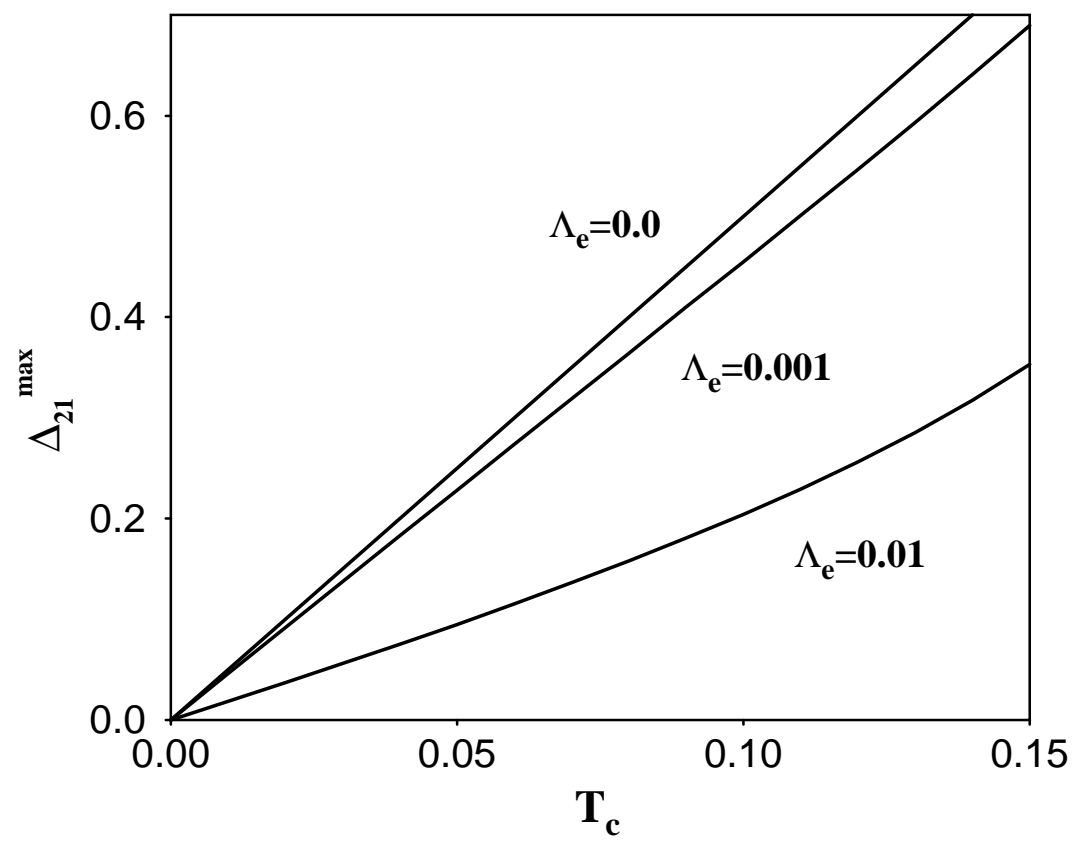

FIG. 3. Refrigeration window as a function of $T_{c}$ for 3 values of the coupling coefficient with the environmental bath. Other system parameters are fixed at: $T_{h}=T_{e}=0.2, \Delta_{31}=1$, $\Lambda_{h}=\Lambda_{c}=\epsilon=0.001$ and $s_{c}=1$. 


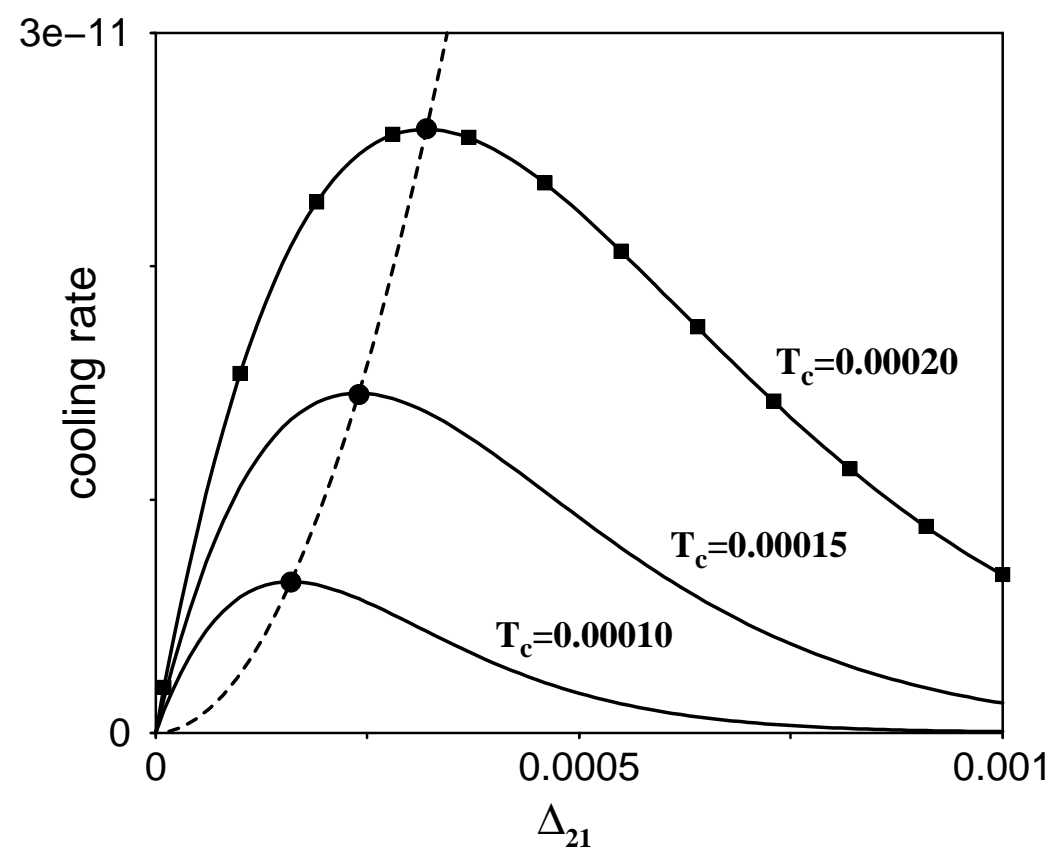

FIG. 4. Cooling rate as a function of $\Delta_{21}$ for 3 values of cold bath temperature. Other system parameters are fixed at: $T_{h}=T_{e}=0.03, \Delta_{31}=1, \Lambda_{h}=\Lambda_{c}=\Lambda_{e}=\epsilon=0.001$ and $s_{c}=1$. The broken curve and solid circles indicate the locus of maximum cooling rate. The solid curves are nominally exact numerical results, while the solid squares illustrate the accuracy of the approximation of Eq (5.2).

Solving for the maximum cooling rate numerically in the limit $T_{c} \rightarrow 0$ reveals that this 3-level quantum chiller respects the fundamental bound of Eq (1.1). A more satisfying analytic derivation is possible once we establish the accuracy of an approximate closed-form expression for the cooling rate in the limit of the absolute zero. In the limit of vanishing $T_{c}$ : (a) $\Delta_{21}^{\max }$ vanishes; (b) $\Delta_{32} \approx \Delta_{31}$; and (c) the coefficients $c_{10}, c_{20}$ and $c_{d 0}$ of Appendix $\mathbb{B}$ become independent of both $T_{c}$ and $\Delta_{21}$. Then, to an excellent approximation, the cooling rate reduces to

$$
\dot{\mathcal{Q}}_{c} \approx \Delta_{21}^{\left(s_{c}+1\right)} \frac{\Lambda_{c}}{1-e^{-\Delta_{21} / T_{c}}} \frac{\left(e^{-\Delta_{21} / T_{c}} c_{10}-c_{20}\right)}{c_{d 0}} .
$$

The accuracy of this approximation is depicted in Fig 4 . Although maximizing the cooling rate of $\mathrm{Eq}(5.2)$ yields a transcendental equation that can only be solved numerically, the solution for that transition energy $\Delta_{21}^{*}$ is of the form $\Delta_{21}^{*} \propto T_{c}$, independent of the coupling 
with the environmental bath or the value of the positive exponent $s_{c}$ in Eq (4.4). It then follows from $\mathrm{Eq}(5.2)$ that

$$
\dot{\mathcal{Q}}_{c}^{\max } \propto T_{c}^{\left(s_{c}+1\right)} \quad\left(s_{c}>0\right)
$$

as required by the Second and Third laws [1].

\section{Efficiency}

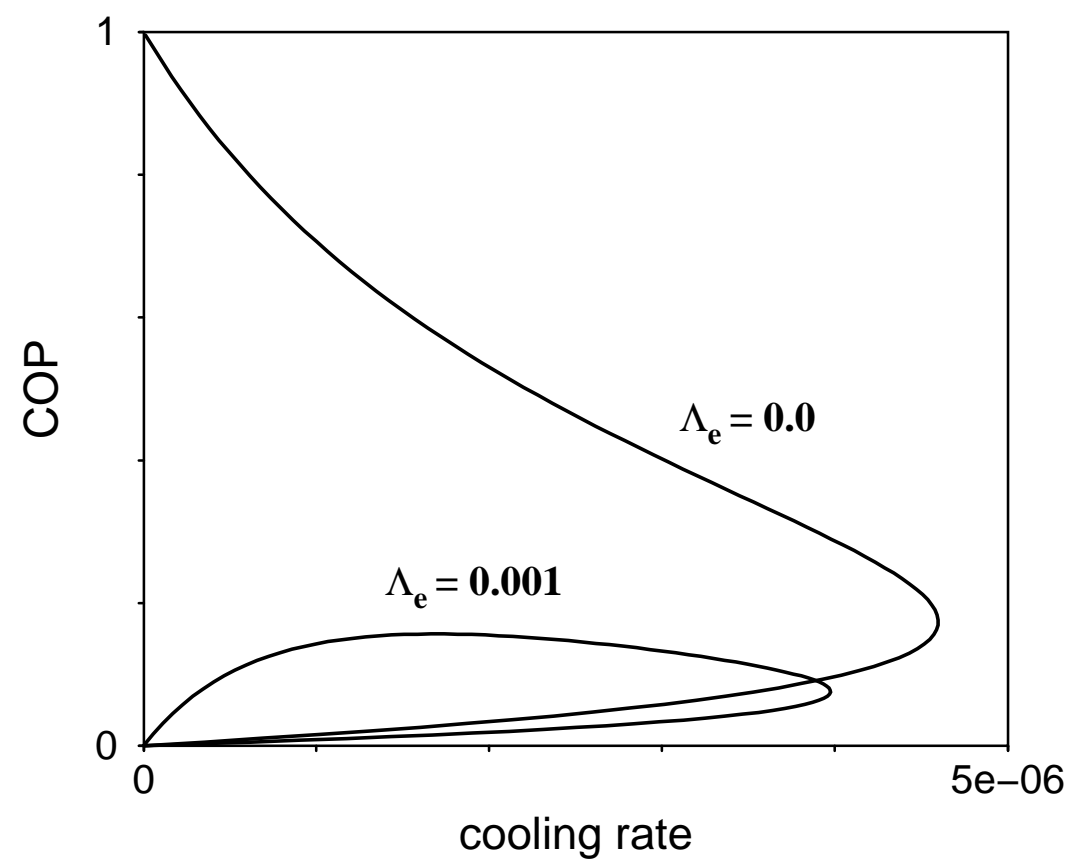

FIG. 5. Characteristic chiller plot, COP against cooling rate, with and without coupling to the environmental bath. The control variable is $\Delta_{21}$. System parameters are fixed at: $T_{c}=0.1$, $T_{h}=T_{e}=0.2, \Delta_{31}=1, \Lambda_{h}=\Lambda_{c}=\epsilon=0.001$ and $s_{c}=1$.

Cooling cycle efficiency is usually defined by Coefficient of Performance (COP), the ratio of cooling rate to input power, which in this instance is $\mathrm{COP}=\dot{\mathcal{Q}}_{c} / \dot{\mathcal{W}}$. Cooling cycles are conveniently characterized by a plot of COP against cooling rate [13], as in Fig 5. Even in the absence of the (parasitic) environmental bath, the 3-level system possesses an energy leak that militates against efficient operation as $\Delta_{21} \rightarrow 0$ [1], which appears as the lower 
branch of the curves in Fig 5, with COP vanishing as cooling rate is lowered. At the other end of the refrigeration window $\left(\Delta_{21} \rightarrow \Delta_{21}^{\max }\right)$, the existence of the extra dissipation to the environmental bath makes a quantum difference. In the absence of this extra energy-leak pathway, there are no irreversibilities that mitigate against efficiency operation, so both cooling rate and power input vanish at the same rate such that the COP approaches its fundamental reversible (Carnot) value of $T_{c} /\left(T_{h}-T_{c}\right)$ [1, 13]. The coupling to the environmental bath introduces a loss mechanism that mitigates against this nominally slow operation, so $\mathrm{COP}$ also vanishes in this limit (the upper branch of the curve in Fig 5 for $\Lambda_{e}>0$ ). Fig 6 offers an alternative view of the differences in dissipation: a plot of entropy production rate against $\Delta_{21}$ with and without coupling to the environmental bath.

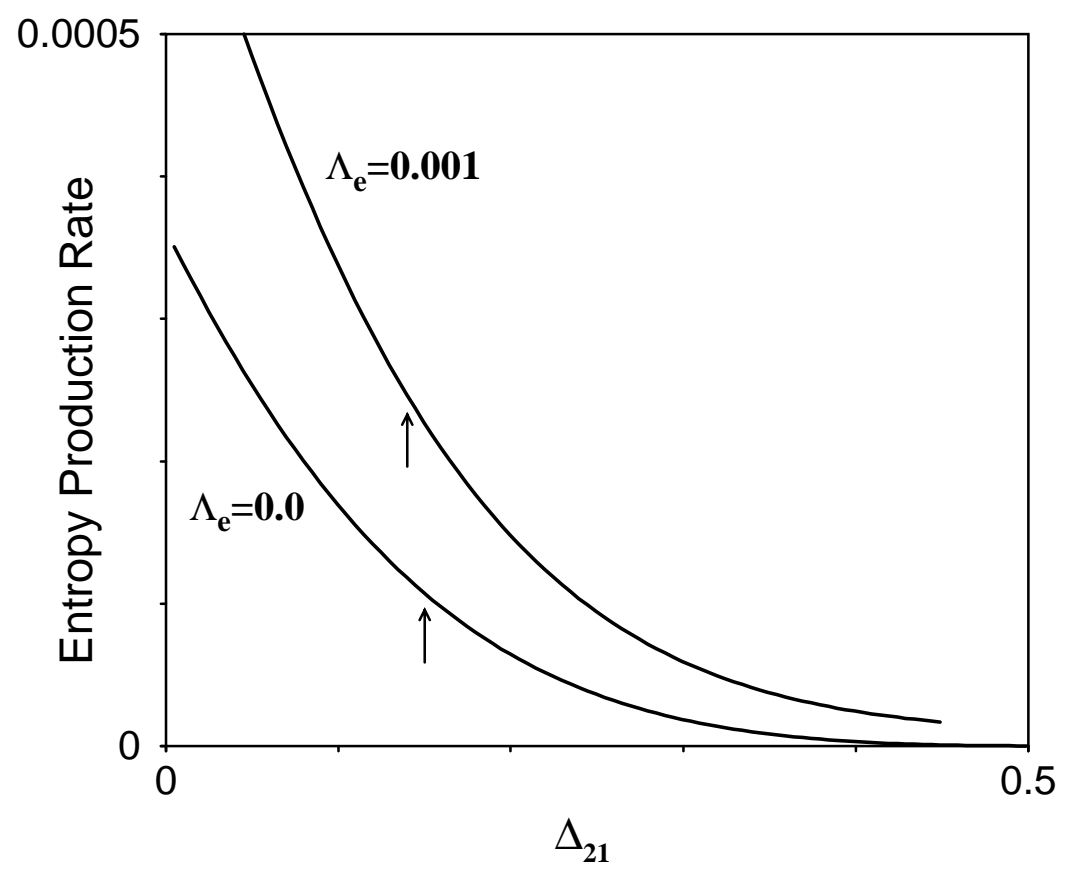

FIG. 6. Entropy production rate as a function of $\Delta_{21}$, with and without coupling to the environmental bath. The refrigeration window is broader in the absence of this coupling (the two curves terminate at different values of $\left.\Delta_{21}^{\max }\right)$. Arrows indicate the points of maximum cooling rate. System parameters are fixed at: $T_{c}=0.1, T_{h}=T_{e}=0.2, \Delta_{31}=1, \Lambda_{h}=\Lambda_{c}=\epsilon=0.001$ and $s_{c}=1$. 


\section{QUANTUM ABSORPTION CHILLER}

Classical cooling cycles can be driven by thermal sources that are hotter than the heat rejection reservoir, commonly called absorption chillers (in contrast to more common workdriven mechanical chillers) [13]. A simple quantum analog is our 3-level cooling cycle with $T_{e}>T_{h}$, where the environmental bath is analogous to what is called the generator in classical absorption cycles. Consider de-coupling the 3-level system from the coherent radiation field and driving it with incoherent radiation (e.g., a flash lamp) at input thermal power $\dot{\mathcal{Q}}_{e}$. The environmental bath becomes a heat source rather than a dissipative sink. As in classical absorption chillers, the COP is defined as $\dot{\mathcal{Q}}_{c} / \dot{\mathcal{Q}}_{e}$. While the refrigeration window and characteristic chiller curve will now be derived, we note that the basic result for the dependence of maximum cooling rate on $T_{c}$ in the limit $T_{c} \rightarrow 0$ (5.3) remains unaltered.

The refrigeration window follows from $\mathrm{Eq}(3.5)$ :

$$
\Delta_{21}^{\max }=\frac{T_{c}\left(T_{e}-T_{h}\right)}{T_{h}\left(T_{e}-T_{c}\right)} \Delta_{31}
$$

which, as for the dissipative environmental bath at $T_{e} \leq T_{h}$, vanishes in the limit $T_{c} \rightarrow 0$.

The characteristic chiller curve is graphed in Fig 7. With $T_{e}>T_{h}$, there is no irreversibil-

ity that undermines efficient slow operation $\left(\Delta_{21} \rightarrow \Delta_{21}^{\max }\right)$. Hence the ordinate intercept of each curve can approach the reversible Carnot limit for absorption cycles of [13]

$$
\mathrm{COP}_{\text {Carnot }}=\frac{\frac{1}{T_{h}}-\frac{1}{T_{e}}}{\frac{1}{T_{c}}-\frac{1}{T_{h}}} .
$$




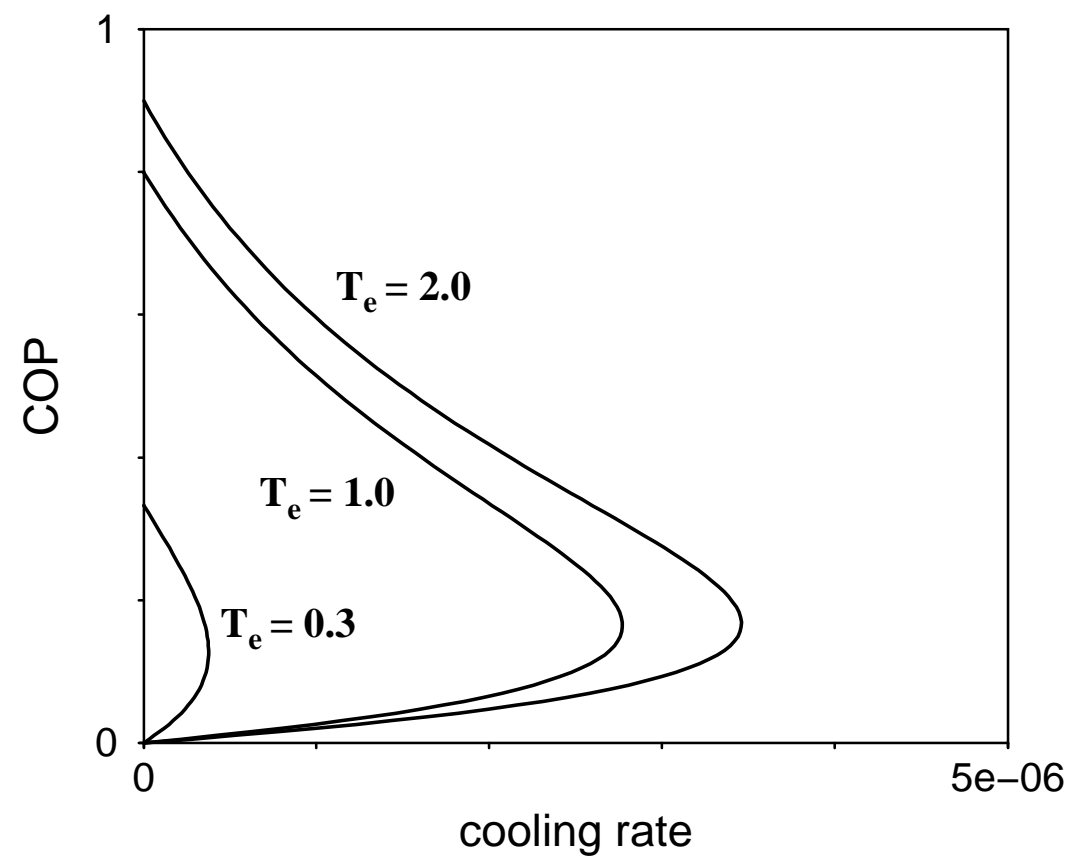

FIG. 7. COP against cooling rate for the 3-level quantum absorption cycle, at 3 values of $T_{e}$. System parameters are fixed at: $T_{c}=0.1, T_{h}=0.2, \Delta_{31}=1, \Lambda_{h}=\Lambda_{c}=\Lambda_{e}=\epsilon=0.001$ and $s_{c}=1$.

Sections $\mathrm{V}$ and $\mathrm{VI}$ addressed the extreme cases of the power input to the cooling cycle being either pure coherent radiation or purely incoherent and thermal, respectively. A combination of the two (when $T_{e}>T_{h}$ ) can be calculated directly from the results derived above.

\section{SUMMARY}

In approaching the absolute zero, any cooling cycle must be dominated by quantum dynamics. At the same time, one should be able to probe its behavior with fundamental chiller thermodynamics. The 3-level quantum model proposed and explored here provides a simple case study. It steps beyond earlier quantum refrigeration models in accounting fully for spontaneous emission (and spontaneous absorption), and including an environmental bath that either mimics actual rate-dependent dissipative mechanisms in work-driven chillers when $T_{e} \leq T_{h}$, or establishes the quantum analog of an absorption (purely thermally-driven) 
chiller when $T_{e}>T_{h}$. All the irreversibilities modeled here and in previous studies [1,2] are effectively heat leaks. The quantum analog of internal friction stems from the noncommutation of the field and system Hamiltonians. In the limit of week fields $(\epsilon \rightarrow 0)$, this internal dissipation grows negligible. Incorporating the quantum version of friction remains a challenge for a future study.

Subject to reasonable approximations that retain the integrity of the system and its reservoirs, expressions can be derived for: (a) the conditions under which a cooling effect can be generated, (b) chiller efficiency as a function of cooling rate, and (c) the temperature dependence of the fastest rate at which molecular systems can be cooled at ultra-low temperatures. The relation of this maximum cooling rate to the energy mode density has been established, and the basic result has been shown to respect the fundamental bound that was recently established for maximum cooling rate from the Second and Third Laws.

\section{Acknowledgments}

J.P.P. acknowledges a Golda Meier Fellowship from the Hebrew University of Jerusalem. Work supported by the Israel Science Foundation. The Fritz Haber Research Center is supported by the Minerva Gesellschaft für die Forschung, GmbH München, Germany.

\section{APPENDIX A: QUANTUM REDUCED DYNAMICS}

To obtain the evolution equation for the 3-level system, we invoke standard tools for quantum open systems: the Redfield approach [14,15] and the secular approximation [11,16]. Assuming (a) weak coupling of the system with its bath, (b) a weak driving field, (c) rapidly decaying bath correlation functions, and (d) an initial density matrix in the form of Eq (2.5), we can express the evolution equation as:

$$
\begin{gathered}
\dot{\hat{\rho}}_{s}(t)=-i\left[\hat{H}_{s}+\hat{H}_{s f}(t), \hat{\rho}_{s}(t)\right]+ \\
\left\{-\bar{G}_{\hat{\Gamma}_{h} \hat{\Gamma}_{h}^{\dagger}}\left(\Delta_{31}\right)\left[\hat{P}_{31}, \hat{P}_{13} \hat{\rho}_{s}(t)\right]-\bar{G}_{\hat{\Gamma}_{h}^{\dagger} \hat{\Gamma}_{h}}\left(-\Delta_{31}\right)\left[\hat{P}_{13}, \hat{P}_{31} \hat{\rho}_{s}(t)\right]\right\}+\{\text { H.C. }\}+ \\
\left\{-\bar{G}_{\hat{\Gamma}_{c} \hat{\Gamma}_{c}^{\dagger}}\left(\Delta_{21}\right)\left[\hat{P}_{21}, \hat{P}_{12} \hat{\rho}_{s}(t)\right]-\bar{G}_{\hat{\Gamma}_{c}^{\dagger} \hat{\Gamma}_{c}}\left(-\Delta_{21}\right)\left[\hat{P}_{12}, \hat{P}_{21} \hat{\rho}_{s}(t)\right]\right\}+\{\text { H.C. }\}+
\end{gathered}
$$




$$
\left\{-\bar{G}_{\hat{\Gamma}_{e} \hat{\Gamma}_{e}^{\dagger}}\left(\Delta_{32}\right)\left[\hat{P}_{32}, \hat{P}_{23} \hat{\rho}_{s}(t)\right]-\bar{G}_{\hat{\Gamma}_{e}^{\dagger} \hat{\Gamma}_{e}}\left(-\Delta_{32}\right)\left[\hat{P}_{23}, \hat{P}_{32} \hat{\rho}_{s}(t)\right]\right\}+\{\text { H.C. }\}
$$

where $\{$ H.C. $\}$ denotes the Hermitian conjugate of the expression in brackets that immediately precedes it. Eq (A1) is valid independent of the nature of the baths as long as the coupling is weak and the bath correlation functions decay quickly.

The influence of the baths is included in the coefficients $\bar{G}$,

$$
\bar{G}_{\hat{\Gamma}_{b} \hat{\Gamma}_{b}^{\prime}}\left(\Delta_{b}\right)=\int_{0}^{\infty} d t e^{i t \Delta_{b}} C_{\hat{\Gamma}_{b} \hat{\Gamma}_{b}^{\prime}}
$$

with $\Delta_{b}=\Delta_{31}, \Delta_{21}, \Delta_{32}$ for $b=h, c, e$, respectively, and $C_{\hat{\Gamma}_{b} \hat{\Gamma}_{b}^{\prime}}$ being given by (2.7). When $\hat{\Gamma}_{b}^{\prime}=\hat{\Gamma}_{b}^{\dagger}, \bar{G}$ can be decomposed as

$$
\bar{G}_{\hat{\Gamma}_{b} \hat{\Gamma}_{b}^{\dagger}}\left(\Delta_{b}\right)=\frac{\bar{C}_{\hat{\Gamma}_{b} \hat{\Gamma}_{b}^{\dagger}}\left(\Delta_{b}\right)}{2}+i \bar{S}_{\hat{\Gamma}_{b} \hat{\Gamma}_{b}^{\dagger}}\left(\Delta_{b}\right)
$$

with $\bar{C}, \bar{S}$ being real and $\bar{C} \geq 0$ [9,15, 17]. With Eq (बत3), we split each term of Eq (A1) into two parts related to $\bar{C}$ and $\bar{S}$. The $\bar{C}$-related term is

$$
-\frac{\bar{C}_{\hat{\Gamma}_{b} \hat{\Gamma}_{b}^{\dagger}}\left(\Delta_{b}\right)}{2}\left\{\left[\hat{P}_{b}, \hat{P}_{b}^{\dagger} \hat{\rho}_{s}(t)\right]+\left[\hat{\rho}_{s}(t) \hat{P}_{b}, \hat{P}_{b}^{\dagger}\right]\right\}-\frac{\bar{C}_{\hat{\Gamma}_{b}^{\dagger} \hat{\Gamma}_{b}}\left(-\Delta_{b}\right)}{2}\left\{\left[\hat{P}_{b}^{\dagger}, \hat{P}_{b} \hat{\rho}_{s}(t)\right]+\left[\hat{\rho}_{s}(t) \hat{P}_{b}^{\dagger}, \hat{P}_{b}\right]\right\}
$$

with $\hat{P}_{b}=\hat{P}_{31}, \hat{P}_{21}, \hat{P}_{32}$ for $b=h, c, e$ respectively. It is readily confirmed that the terms of (A4) have the standard Lindblad form that insures complete positivity of the dynamics.

For simplicity of notation in Sections $\mathrm{M} \mathbb{\square}$ and Appendix B, we introduce the notation

$$
\lambda_{b}=\lambda_{b}\left(\Delta_{b}, T_{b}\right)=\bar{C}_{\hat{\Gamma}_{b} \hat{\Gamma}_{b}^{\dagger}}\left(\Delta_{b}\right), \quad \bar{\lambda}_{b}=\bar{\lambda}_{b}\left(\Delta_{b}, T_{b}\right)=\bar{C}_{\hat{\Gamma}_{b}^{\dagger} \hat{\Gamma}_{b}}\left(-\Delta_{b}\right)
$$

with $\lambda_{b}$ and $\hat{\lambda}_{b}$ related by

$$
\bar{\lambda}_{b}=e^{-\Delta_{b} / T_{b}} \lambda_{b}
$$

The $\bar{S}$-related term is

$$
i \bar{S}_{\hat{\Gamma}_{b} \hat{\Gamma}_{b}^{\dagger}}\left(\Delta_{b}\right)\left[\hat{P}_{b} \hat{P}_{b}^{\dagger}, \hat{\rho}_{s}(t)\right]+i \bar{S}_{\hat{\Gamma}_{b}^{\dagger} \hat{\Gamma}_{b}}\left(-\Delta_{b}\right)\left[\hat{P}_{b}^{\dagger} \hat{P}_{b}, \hat{\rho}_{s}(t)\right]
$$


$\hat{P}_{b} \hat{P}_{b}^{\dagger}$ and $\hat{P}_{b}^{\dagger} \hat{P}_{b}$ represent small corrections to the system's energy levels, which in the weak coupling limit also turn out to be negligible.

Hence, from Eq (A1), the time evolution equation for any operator (in the Heisenberg representation) can be obtained, and the germane results are provided in Section $\mathbb{1}$.

\section{APPENDIX B: COEFFICIENTS AT STEADY STATE}

The coefficients $p_{i j}$ are the solution of a $4 \times 4$ system of linear equations the coefficients of which depend on: (a) the system energy structure, (b) the coupling with the field $\epsilon$, and (c) the coupling with the baths $\lambda_{b}$ and $\bar{\lambda}_{b}$ :

$$
\begin{aligned}
& p_{11}=\left(c_{10}+c_{11} \lambda_{c}+c_{12} \lambda_{c}^{2}\right) / D \\
& p_{22}=\left(c_{20}+c_{21} \lambda_{c}+\bar{c}_{21} \bar{\lambda}_{c}+\bar{c}_{22} \lambda_{c} \bar{\lambda}_{c}\right) / D \\
& p_{33}=\left(c_{30}+c_{31} \lambda_{c}+\bar{c}_{31} \bar{\lambda}_{c}+c_{32} \lambda_{c}^{2}+\bar{c}_{32} \lambda_{c} \bar{\lambda}_{c}\right) / D \\
& p_{32}=i\left(c_{c 0}+c_{c 1} \lambda_{c}+\bar{c}_{c 1} \bar{\lambda}_{c}\right) / D, \quad p_{23}=p_{32}^{*}
\end{aligned}
$$

where the denominator $D$ is

$$
D=c_{d 0}+c_{d 1} \lambda_{c}+\bar{c}_{d 1} \bar{\lambda}_{c}+c_{d 2} \lambda_{c}^{2}+\bar{c}_{d 2} \lambda_{c} \bar{\lambda}_{c}
$$

and the $c$ coefficients are given by

$$
\begin{aligned}
& c_{d 0}=\left(\lambda_{h}+\lambda_{e}+\bar{\lambda}_{e}\right)\left\{\left(\lambda_{h}+\bar{\lambda}_{h}\right) \bar{\lambda}_{e}+\lambda_{e} \bar{\lambda}_{h}\right\}+4 \epsilon^{2}\left(\lambda_{h}+2 \bar{\lambda}_{h}\right) \\
& c_{d 1}=\left(\lambda_{h}+\lambda_{e}+\bar{\lambda}_{e}\right)\left(\lambda_{h}+\bar{\lambda}_{h}+\lambda_{e}\right)+\left(\lambda_{h}+\bar{\lambda}_{h}\right) \bar{\lambda}_{e}+\lambda_{e} \bar{\lambda}_{h}+4 \epsilon^{2} \\
& \bar{c}_{d 1}=\left(\lambda_{h}+\lambda_{e}+\bar{\lambda}_{e}\right)^{2}+8 \epsilon^{2} \\
& c_{d 2}=\lambda_{h}+\bar{\lambda}_{h}+\lambda_{e} \\
& \bar{c}_{d 2}=\lambda_{h}+\lambda_{e}+\bar{\lambda}_{e} \\
& c_{10}=\left(\lambda_{h}+\lambda_{e}+\bar{\lambda}_{e}\right) \lambda_{h} \bar{\lambda}_{e}+4 \epsilon^{2} \lambda_{h} \\
& c_{11}=\left(\lambda_{h}+\lambda_{e}+\bar{\lambda}_{e}\right)\left(\lambda_{h}+\lambda_{e}\right)+\lambda_{h} \bar{\lambda}_{e}+4 \epsilon^{2} \\
& c_{12}=\left(\lambda_{h}+\lambda_{e}\right)
\end{aligned}
$$




$$
\begin{aligned}
& c_{20}=\left(\lambda_{h}+\lambda_{e}+\bar{\lambda}_{e}\right) \lambda_{e} \bar{\lambda}_{h}+4 \epsilon^{2} \bar{\lambda}_{h} \\
& c_{21}=\lambda_{e} \bar{\lambda}_{h} \\
& \bar{c}_{21}=\left(\lambda_{h}+\lambda_{e}+\bar{\lambda}_{e}\right)\left(\lambda_{h}+\lambda_{e}\right)+4 \epsilon^{2} \\
& \bar{c}_{22}=\left(\lambda_{h}+\lambda_{e}\right) \\
& c_{30}=\left(\lambda_{h}+\lambda_{e}+\bar{\lambda}_{e}\right) \bar{\lambda}_{e} \bar{\lambda}_{h}+4 \epsilon^{2} \bar{\lambda}_{h} \\
& c_{31}=\left(\lambda_{h}+\lambda_{e}+\bar{\lambda}_{e}\right) \bar{\lambda}_{h}+\bar{\lambda}_{e} \bar{\lambda}_{h} \\
& \bar{c}_{31}=\left(\lambda_{h}+\lambda_{e}+\bar{\lambda}_{e}\right) \bar{\lambda}_{e}+4 \epsilon^{2} \\
& c_{32}=\bar{\lambda}_{h} \\
& \bar{c}_{32}=\bar{\lambda}_{e} \\
& c_{c 0}=2 \epsilon \bar{\lambda}_{h}\left(\lambda_{e}-\bar{\lambda}_{e}\right) \\
& c_{c 1}=-2 \epsilon \bar{\lambda}_{h} \\
& \bar{c}_{c 1}=2 \epsilon\left(\lambda_{h}+\lambda_{e}-\bar{\lambda}_{e}\right) .
\end{aligned}
$$

The coefficients $A, B$ and $C$ of Eq (3.5) can be expressed as

$$
\begin{aligned}
& A=4 \epsilon^{2} \lambda_{h} \lambda_{c} / D \\
& B=4\left(\bar{\lambda}_{h}+\bar{\lambda}_{c}\right)\left(\lambda_{e}-\bar{\lambda}_{e}\right) / D \\
& C=\lambda_{h} \lambda_{c} \lambda_{e}\left(\lambda_{h}+\lambda_{c}+\lambda_{e}+\bar{\lambda}_{e}\right) / D .
\end{aligned}
$$

[1] R. Kosloff, E. Geva and J. M. Gordon, J. Appl. Phys. 87, 8093 (2000).

[2] E. Geva and R. Kosloff, J. Chem. Phys 104, 7681 (1996).

[3] R. I. Epstein, M. I. Buchwald, B. C. Edwards, T. R. Gosnell and C. E. Mungan, Nature 377, 500 (1995); C. E. Mungan, M. I. Buchwald, B. C. Edwards, R. I. Epstein and T. R. Gosnell, Phys. Rev. Lett. 78, 1030 (1997); C. W. Hoyt, M. Sheik-Bahae, R. I. Epstein, B. C. Edwards and J. E. Anderson, Phys. Rev. Lett. 85, 3600 (2000). 
[4] J. L. Clark, O. F. Miller and G. Rumbles, J. Phys. Chem. A 102, 4428 (1998).

[5] G. S. Argawall and S. Menon, Phys. Rev. A 63, 023818 (2001).

[6] C. Cohen-Tannoudji, J. Dupont-Roc and G. Grynberg, Atom-Photon Interactions (Wiley Interscience, New York, 1992).

[7] G. Lindblad, J. Phys. A: Math. Gen. 29, 4197 (1996).

[8] R. Alicki, J. Phys. A: Math. Gen. 12, L103 (1979).

[9] E. Geva, R. Kosloff and J. L. Skinner, J. Chem. Phys 102, 8541 (1995).

[10] N. V. Prokof'ev and P. C. E. Stamp, Rep. Prog. Phys. 63, 669 (2000).

[11] N. G. van Kampen, Stochastic Processes in Physics and Chemistry (North-Holland, Amsterdam, 1997).

[12] A. J. Legget, S. Chakravarty, A. T. Dorsey, M. P. A. Fisher, A. Garg and W. Zweger, Rev. Mod. Phys. 59, 1 (1987).

[13] J. M. Gordon and K. C. Ng, Cool Thermodynamics (Cambridge International Science Publishing, 2000).

[14] A. G. Redfield, IBM J. 19, 1 (1957).

[15] B. B. Laird, J. Budimir and J. L. Skiner, J. Chem. Phys. 94, 4391 (1991).

[16] E. B. Davies, Commun. Mathem. Phys. 39, 91 (1974).

[17] R. Alicki and K. Lendi, Quantum Dynamical Semigroups and Applications (Springer-Verlag, Berlin 1987). 